Case Report

\title{
Superior Mesenteric Artery Syndrome Associated with Rapid Weight Loss Attributed to Amphetamine Abuse
}

\author{
Richard M. Fazio, ${ }^{1}$ On Chen, ${ }^{2}$ and Wael Eldarawy ${ }^{3}$ \\ ${ }^{1}$ Department of Internal Medicine, Maimonides Medical Center, 4802 Tenth Avenue, Brooklyn, NY 11219, USA \\ ${ }^{2}$ Department of Cardiology, Maimonides Medical Center, Brooklyn, NY, USA \\ ${ }^{3}$ Department of Gastroenterology, NY Methodist Hospital, 506 Sixth Street, Brooklyn, NY 11215, USA
}

Correspondence should be addressed to Richard M. Fazio; rfazio02@gmail.com

Received 24 April 2015; Accepted 3 August 2015

Academic Editor: Daniel C. Damin

Copyright (C) 2015 Richard M. Fazio et al. This is an open access article distributed under the Creative Commons Attribution License, which permits unrestricted use, distribution, and reproduction in any medium, provided the original work is properly cited.

Superior mesenteric artery (SMA) syndrome arises from a reduction in the angle formed between the SMA and the aorta, thereby compressing the third portion of the duodenum. This phenomenon may be caused by a number of factors, one of which being acute weight loss. We report a case of a female patient presenting with abdominal pain and vomiting who developed superior mesenteric artery (SMA) syndrome as a result of rapid weight loss, thought to be secondary to amphetamine abuse. This association can often be overlooked and, to our knowledge, has not been previously reported.

\section{Introduction}

The superior mesenteric artery (SMA) syndrome, also known as Cast Syndrome, Mesenteric Root Syndrome, or Wilke's disease, is a rare cause of mechanical small intestinal obstruction and classically associated with rapid weight loss. SMA syndrome stems from compression on the third portion of the duodenum, resulting from the acute angle created between the superior mesenteric artery and the aorta, as caused by paucity of mesenteric and retroperitoneal fat pads. An illustration of this concept can be seen in Figure 1.

\section{Case Report}

The patient being presented is a 30-year-old Asian-American female with a past medical history significant for Attention Deficit Hyperactivity Disorder (ADHD) and bipolar disorder and is prescribed a daily regimen of $10 \mathrm{mg}$ amphetamine/dextroamphetamine. She presented with right upper quadrant and epigastric pain of 5 days' duration. The pain was described as sharp, constant, and exacerbated by eating. It was associated with bilious, nonbloody vomiting, nausea, anorexia, and a thirty-pound weight loss over a time period of two months. The patient stated that she was under a significant amount of stress and she recently increased the dosage of her amphetamine/dextroamphetamine, the exact amount of which she was not able to specify. Examination revealed cachexia (BMI of 15.6) and right upper quadrant tenderness upon palpation. Abdominal CT scan (Figure 2) revealed a markedly dilated stomach and duodenum and paucity of intraperitoneal fat. Mesenteric angiography (Figure 3) showed a 9-degree angle between the SMA and aorta (normal, 25-60 degrees) and an aortomesenteric distance of $3.6 \mathrm{~mm}$. (normal, $10-28 \mathrm{~mm}$ ) [1-4]. The patient was treated with nasogastric intubation for gastric decompression, total parenteral nutrition, and antiemetic medication to prevent further vomiting episodes. Incremental advancements of enteral feeds were introduced to prevent electrolyte changes seen in refeeding syndrome and the concept of positional feeding (i.e., having the patient in a prone or left lateral decubitus position during feeding) was strongly encouraged. Symptoms continuously improved through her hospitalization, and she was eventually discharged with the ability to tolerate oral feeding. The patient was seen by representatives of the psychiatric department while an inpatient and was scheduled for outpatient follow-up with eventual adjustment 


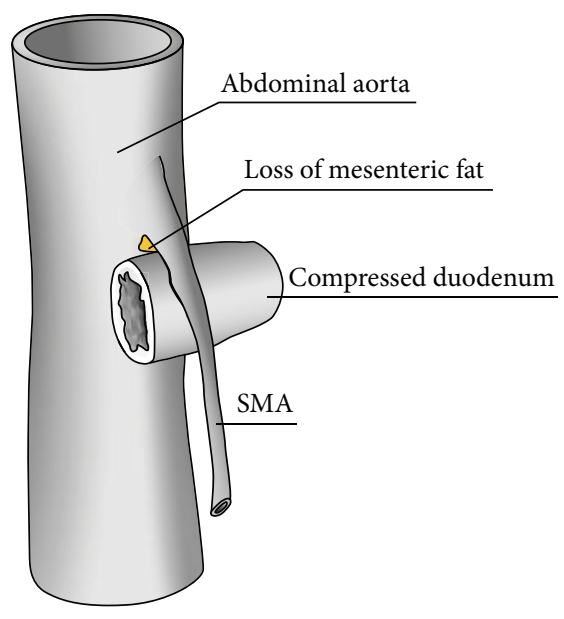

(a)

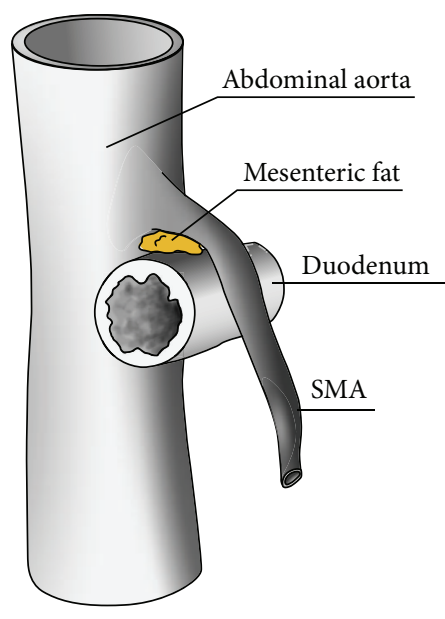

(b)

Figure 1: A schematic view of the aorta, SMA, and third portion of the duodenum. (a) Loss of mesenteric fat producing an acute angle between aorta and SMA and compression of the duodenum. (b) Presence of mesenteric fat and a normal angle between aorta and SMA.

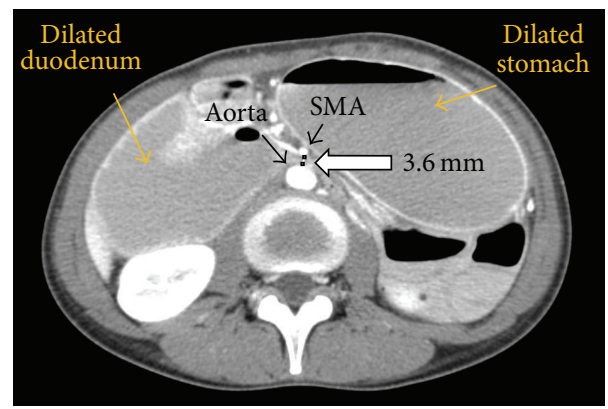

FIGURE 2: Contrast CT of the abdomen showing the distance between aorta and SMA (white arrow, marked as $3.6 \mathrm{~mm}$ ). Also seen are a dilated stomach and duodenum and location of the SMA (black arrow).

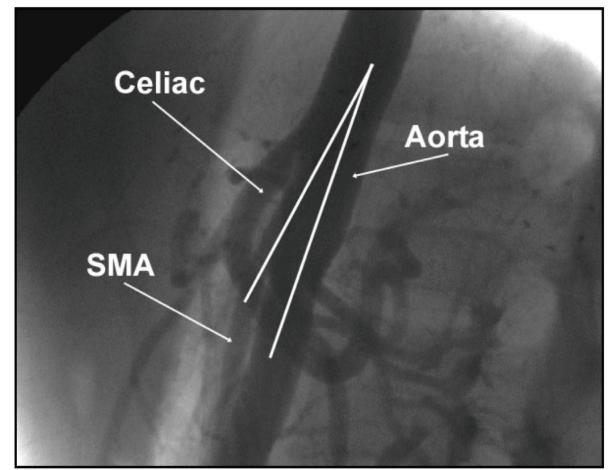

FIGURE 3: Angiography of the abdominal aorta. The celiac and SMA branches are identified, as well as a narrow-angle formed between the abdominal aorta and the SMA branch.

of home medications and discontinuation of amphetamines. Eight months later, she presented to the outpatient medical clinic having reported appropriate weight gain without further symptoms.

\section{Discussion}

Located in the retroperitoneum, the third portion of the duodenum runs through the acute angle created by the aorta and its branching artery known as the superior mesenteric artery $[5,6]$. The normal aortomesenteric angle ranges from 25 to 65 degrees due to the retroperitoneal fat pads that act as a cushion, preventing the SMA from compressing the duodenum. The mean aortomesenteric distance, which is directly proportional to the amount of "cushion" available, measures approximately $10-28 \mathrm{~mm}$ in a healthy person [2$4,6,7]$. Aortomesenteric angles less than 22-25 degrees and distances less than $8 \mathrm{~mm}$ have correlated well with the diagnosis of SMA syndrome [3, 7]. Predisposing factors include a prolonged supine position, an unusually low origin of the SMA, exaggerated lumbar lordosis, and an abnormally high fixation of the duodenojejunal flexure to the ligament of Treitz. Precipitating factors are those that cause marked weight loss resulting in loss of the retroperitoneal fat pad, such as cancer and burns, anorexia nervosa, IV drug abuse, malabsorption, postoperative state disease, trauma to the spine, and application of a body cast [5, 6, 8, 9]. Symptoms of SMA syndrome are those of duodenal compression, namely, nausea, vomiting, eructation, abdominal pain, early satiety, distension, tenderness, and abnormal bowel sounds [10-12]. They are worsened in the postprandial period and may be alleviated by maneuvers which increase the angle between the SMA and the aorta, including lying in either the prone or left lateral decubitus position and when performing the kneeto-chest maneuver $[5,13,14]$. This feature of postprandial abdominal pain may further compound the problem, as it will lead to continued weight loss and thus perpetual symptoms [14]. The diagnosis of SMA syndrome is oftentimes overlooked due to the broad spectrum of differentials associated with these generalized GI symptoms. Also, other conditions may be found in association with this disease, the most common of which being peptic ulcer disease. Hyperchlorhydria is formed in response to the duodenal distention and gastric 
stasis, which may precipitate the formation of ulcers, along with progressive esophagitis and gastritis [7, 15]. Classically, SMA syndrome is diagnosed via upper GI series or CT imaging with oral contrast, which will illustrate proximal duodenal dilatation, obstruction of the third part of the duodenum, and delay of barium passage into the distal duodenum; however, more modern modalities include SMA angiography to measure the angle between the aorta and the SMA, as well as esophagogastroduodenoscopy to demonstrate compression of the duodenum $[1,2,5,13,14,16]$. Treatment of SMA syndrome begins with a conservative approach, especially for those in the acute setting of the disease. This includes promotion of weight gain either by TPN or by enteric feeding, in order to restore the aortomesenteric angle, correction of fluid, and electrolyte balance, and addressing any underlying precipitating factors $[2,5,10,14]$. Surgical intervention becomes necessary when there is failure following conservative medical therapy and progressive weight loss in the setting of longstanding disease or as a result of complicated peptic ulcer disease $[9,17]$. The goal of a surgical approach is duodenal decompression, which may be achieved through bypass of the obstructed segment of small bowel, as seen in both gastrojejunostomy and duodenojejunostomy techniques or through mobilization of the duodenum, as performed during Strong's procedure. Duodenojejunostomy has become the preferred procedure amongst the surgical community due to its higher success rates and decreased risk for bacterial overgrowth syndrome. In particular, a laparoscopic approach has drawn favor over the traditional open techniques as a way to decrease morbidity, shorten hospital stays, and increase the overall success rate of the procedure $[14,18]$.

\section{Conclusion}

The FDA approves the use of amphetamines for indications such as ADHD and narcolepsy; however, the off-label use and abuse of these drugs have grown to become a dangerous epidemic in the United States in recent years, most notably amongst young, college-aged individuals. Amphetamine abuse may involve acquisition from illicit sources or manipulation of legal doses, which we suspect led to our patient's rapid weight loss and SMA syndrome. Amphetamine abuse may be difficult to recognize, and a patient's history is oftentimes withheld. Therefore, when presented with a patient complaining of abdominal symptoms mimicking small bowel obstruction in the setting of rapid weight loss, one should consider the diagnosis of amphetamine-induced weight loss causing SMA syndrome.

\section{Conflict of Interests}

The authors declare that there is no conflict of interests regarding the publication of this paper.

\section{References}

[1] L. Gustafsson, A. Falk, P. J. Lukes, and R. Gamklou, "Diagnosis and treatment of superior mesenteric artery syndrome," British Journal of Surgery, vol. 71, no. 7, pp. 499-501, 1984.
[2] F. Lippl, C. Hannig, W. Weiss, H.-D. Allescher, M. Classen, and M. Kurjak, "Superior mesenteric artery syndrome: diagnosis and treatment from the gastroenterologist's view," Journal of Gastroenterology, vol. 37, no. 8, pp. 640-643, 2002.

[3] B. Unal, A. Aktaş, G. Kemal et al., "Superior mesenteric artery syndrome: CT and ultrasonography findings," Diagnostic and Interventional Radiology, vol. 11, pp. 90-95, 2005 (Latvian).

[4] J. R. Derrick and H. A. Fadhli, "Surgical anatomy of the superior mesenteric artery," The American Surgeon, vol. 31, pp. 545-547, 1965.

[5] T. Welsch, M. W. Büchler, and P. Kienle, "Recalling superior mesenteric artery syndrome," Digestive Surgery, vol. 24, no. 3, pp. 149-156, 2007.

[6] N. Mathenge, S. Osiro, I. I. Rodriguez, C. Salib, R. S. Tubbs, and M. Loukas, "Superior mesenteric artery syndrome and its associated gastrointestinal implications," Clinical Anatomy, vol. 27, no. 8, pp. 1244-1252, 2014.

[7] M. Stamatakos, K. Kontzoglou, C. Stefanaki et al., "Wilkie syndrome. What is this?" Chirurgia, vol. 104, no. 1, pp. 11-15, 2009.

[8] J. B. Barnes and M. Lee, "Superior mesenteric artery syndrome in an intravenous drug abuser after rapid weight loss," Southern Medical Journal, vol. 89, no. 3, pp. 331-334, 1996.

[9] Y. Gunduz, F. Altintoprak, K. Asil, and G. Cakmak, "An uncommon cause of abdominal pain and weight loss: the superior mesenteric artery syndrome," Vasa, vol. 43, no. 2, pp. 149-153, 2014.

[10] S. A. Jones, R. Carter, L. L. Smith, and E. J. Joergenson, "Arteriomesenteric duodenal compression," The American Journal of Surgery, vol. 100, no. 2, pp. 262-277, 1960.

[11] A. R. Ahmed and I. Taylor, "Superior mesenteric artery syndrome," Postgraduate Medical Journal, vol. 73, no. 866, pp. 776778, 1997.

[12] A. R. Mansberger Jr., J. B. Hearn, R. M. Byers, N. Fleisig, and R. W. Buxton, "Vascular compression of the duodenum. Emphasis on accurate diagnosis," The American Journal of Surgery, vol. 115, no. 1, pp. 89-96, 1968.

[13] J. R. Hines, R. M. Gore, and G. H. Ballantyne, "Superior mesenteric artery syndrome. Diagnostic criteria and therapeutic approaches," The American Journal of Surgery, vol. 148, no. 5, pp. 630-632, 1984.

[14] N. D. Merrett, R. B. Wilson, P. Cosman, and A. V. Biankin, "Superior mesenteric artery syndrome: diagnosis and treatment strategies," Journal of Gastrointestinal Surgery, vol. 13, no. 2, pp. 287-292, 2009.

[15] D. A. Geer, "Superior mesenteric Artery syndrome," Military Medicine, vol. 7, pp. 321-323, 1990.

[16] R. Bedoya, S. M. Lagman, G. P. Pennington, and A. Kirdnual, "Clinical and radiological aspects of the superior mesenteric artery syndrome," The Journal of the Florida Medical Association, vol. 73, no. 9, pp. 686-689, 1986.

[17] U. Baltazar, J. Dunn, C. Floresguerra, L. Schmidt, and W. Browder, "Superior mesenteric artery syndrome: an uncommon cause of intestinal obstruction," Southern Medical Journal, vol. 93, no. 6, pp. 606-608, 2000.

[18] B. J. Pottorf, F. A. Husain, H. W. Hollis, and E. Lin, "Laparoscopic management of duodenal obstruction resulting from superior mesenteric artery syndrome," JAMA Surgery, vol. 149, no. 12, pp. 1319-1322, 2014. 


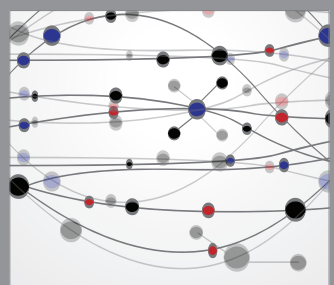

The Scientific World Journal
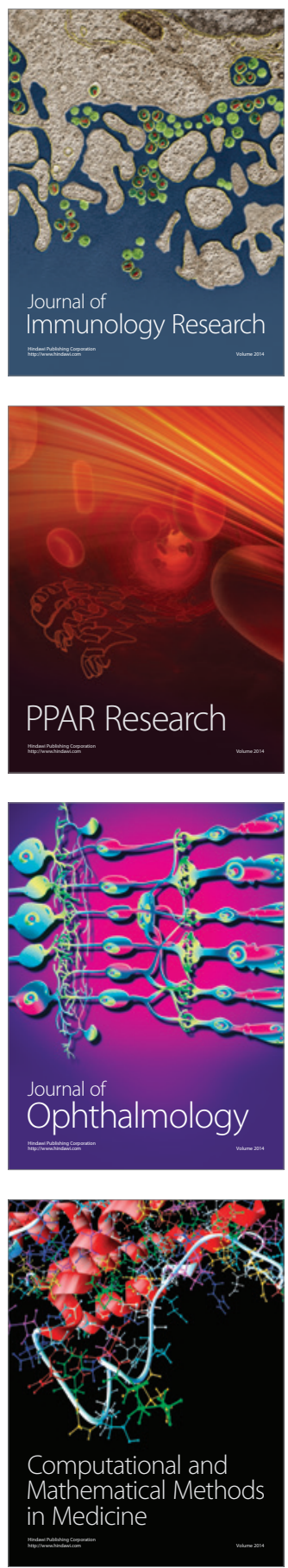

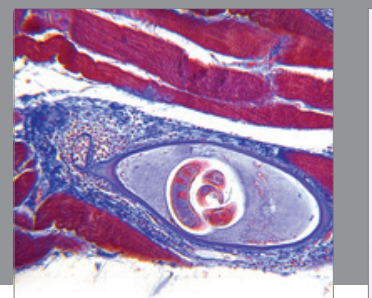

Gastroenterology

Research and Practice
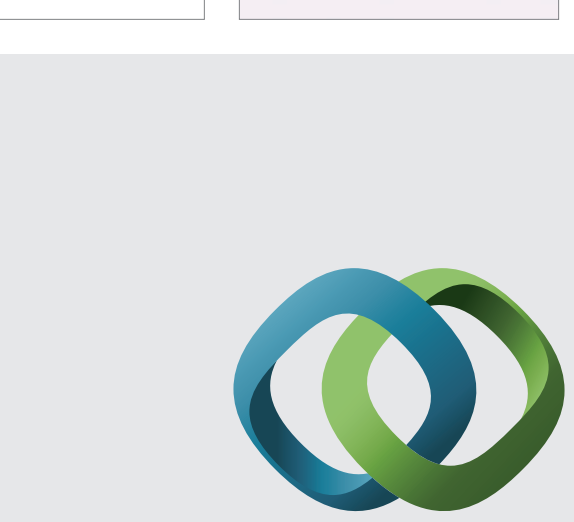

\section{Hindawi}

Submit your manuscripts at

http://www.hindawi.com
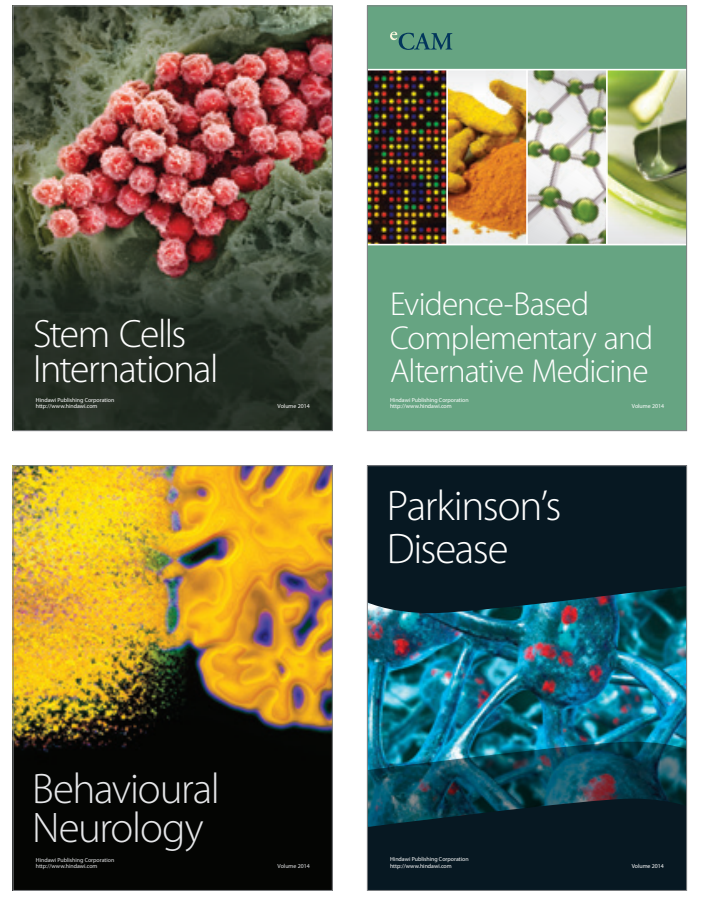
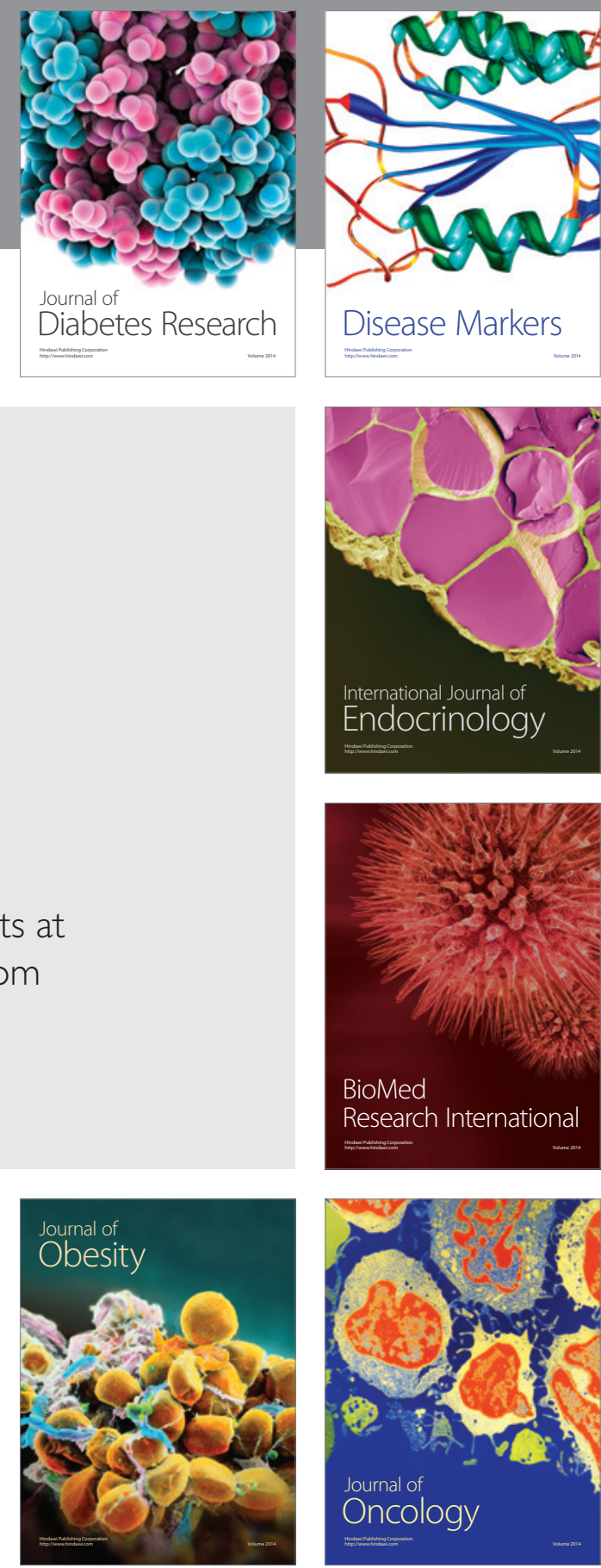

Disease Markers
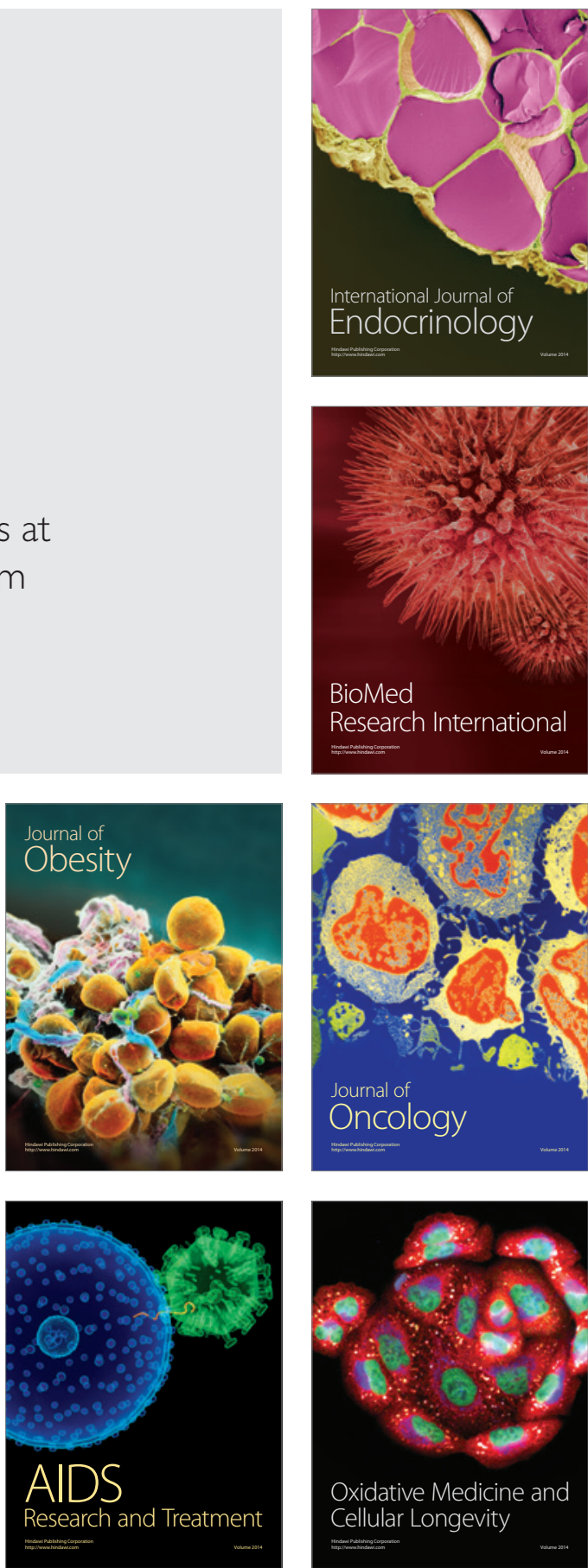